\title{
Assessing the Effect of Wind Farm Layout on Energy Storage Requirement for Power Fluctuation Mitigation
}

\author{
Hessam Kazari, Student Member, IEEE, Hashem Oraee, Senior Member, IEEE, and Bikash C. Pal, Fellow, IEEE
}

\begin{abstract}
Optimization of wind farm (WF) layout has been studied in the literature with the objective of maximizing the wind energy capture. Based on the power spectrum density (PSD) theorem, this paper shows that the WF layout affects not only the total harvested energy but also the level of power fluctuation, which in turn influences required capacity of battery energy storage system (BESS) needed to mitigate the inherent power fluctuation of the wind farms. Since both harvested energy level and BESS capacity directly influence the profit of WF owner, the effect of WF layout on these quantities are taken into account simultaneously and WF layout optimization problem is redefined. Genetic algorithm (GA) is then employed in order to optimize the resulting objective function. The proposed method and optimization process are performed on the layout of an actual offshore WF using real wind data. A new index is introduced to quantify the power fluctuations, and energy curtailment is assessed. The comparative analysis between the actual layout performance and the optimal layout in different scenarios is conducted, showing the reduction of power fluctuations and improvement of energy curtailment. In addition, different BESS technologies have been analyzed to study the impact of their parameters on the optimization results.
\end{abstract}

Index Terms-Power fluctuation, wind farm, battery energy storage, optimal layout, genetic algorithm (GA)

\section{NOMENCLATURE}

$E(\cdot) \quad$ Expected value

$S_{i i} \quad$ PSD function of the measured wind speed at the $i$ th wind turbine

$S_{i j} \quad$ Cross PSD function between turbines $i$ and $j$

$S_{H} \quad$ PSD function of the variation components at high frequency

$S_{L} \quad$ PSD function of the variation components at low frequency

$V_{w 0} \quad$ Mean wind speed at 10-minute interval

$\sigma_{w}^{2} \quad$ Standard deviation of wind speed at 10-minute interval

$z \quad$ Hub height of the wind turbine

$L_{1} \quad$ Length scale

$\alpha_{L}, \beta_{L}$ Constant values of low frequency component function

$\gamma_{i j} \quad$ Coherence function

$a_{m n} \quad$ Decay factor between turbines $m$ and $n$

$d_{m n} \quad$ Distance between turbines $m$ and $n$

$\tau_{m n} \quad$ Wind traverse time from turbine $m$ to turbine $n$

$\alpha_{m n} \quad$ Wind inflow angle between turbines $m$ and $n$

$A_{\text {long }}$ Decay factor with longitudinal wind direction

H. Kazari and H. Oraee are with Sharif University of Technology, Tehran, Iran (e-mail: hkazari@ee.sharif.edu; oraee@sharif.edu).

B. C. Pal is with the Department of Electrical and Electronic Engineering, Imperial College London, UK (e-mail: b.pal@imperial.ac.uk).
$A_{\text {lat }} \quad$ Decay factor with lateral wind direction

$S_{i i, e q}$ Equivalent PSD of wind speed at the $i$ th turbine

$F_{w t, i i} \quad$ Wind turbine admittance function

$P_{t_{i}} \quad$ Power output of the $i$ th turbine

$P_{W F} \quad$ Total power output of the wind farm

$P_{O} \quad$ Aggregated power output of integrated wind farm and storage

$P_{b} \quad$ Power output of BESS

$T_{p e r} \quad$ Length of simulated wind speed time series

$P_{f} \quad$ Forecasted power output of wind farm

$P_{b}^{r} \quad$ Rated power capacity of BESS

$E_{b} \quad$ Stored energy level of BESS

$E_{b}^{r} \quad$ Rated energy capacity of BESS

$\eta_{c}, \eta_{d} \quad$ Efficiency of charging / discharging of battery

$N_{s}, N_{d}$ Number of intervals for speed/direction of wind

$V_{i} \quad$ Wind speed at $i$ th bin

$d_{j} \quad$ Wind direction at $j$ th bin

$N_{i j} \quad$ Number of occurrence of $\left(V_{i}, d_{j}\right)$

$\operatorname{Pr}_{i j} \quad$ Probability of occurrence of $\left(V_{i}, d_{j}\right)$

$P_{b, o p t}$ Optimum power capacity of BESS

$E_{b, o p t}$ Optimum energy capacity of BESS

$P_{\text {annual }}$ Average annual power production of wind farm

$P_{W F, a v}$ Average steady state wind farm power production with wake effect

$C_{B E S S}$ Cost of battery energy storage system

$c_{P}, c_{E}$ Cost coefficients for power/energy capacities of BESS

$I \quad$ Inflation rate

$T_{B E S S}$ Service life span of BESS

$\rho_{W E} \quad$ Price of wind energy sold to the utility company

$d_{\text {min }}$ Minimum distance allowed between turbines

$x_{i}, y_{i} \quad$ Coordinates of $i$ th turbine in $\mathrm{x}-\mathrm{y}$ plane

$X_{T} \quad$ Tilt factor of wind farm layout

$\theta_{r} \quad$ Rotation angle of wind farm layout

$d P_{x} \quad$ Unacceptable power deviation

$N_{x} \quad$ Number of occurrence of unacceptable power deviations

$E_{\text {curt }}$ Curtailed energy

$P_{\text {curt }} \quad$ Curtailed power

\section{INTRODUCTION}

$\mathbf{P}$ OWER fluctuation of wind farms (WF) is considered as one of the major challenges to realize electricity supply through $100 \%$ [1]. Wind power variations are hard to predict with high accuracy. The prediction error has the effect on system operational and control performance including generation dispatch [2], [3]. 
Energy Storage System (ESS) is a viable option for mitigating the impact of wind generation fluctuation and enabling the integrated WF and storage system that can be dispatched in the same manner as that of a conventional generating unit [4]. The concept of an ESS-integrated WF is to store the generated wind energy during high wind speed time intervals and supply the stored energy during low wind speed time intervals. Since ESS is an expensive and energy limited resource, optimizing its capacity and utilizing a proper control strategy are important.

Basically, ESS can be characterized by its energy capacity, power capacity, ramping capability, and round-trip efficiency. The evaluation of the optimal capacity of ESS for wind farm integration has been addressed in numerous papers and various methods are presented [5]-[8]. So far, several approaches have been proposed for integration of ESS into WF. In [5] Fourier analysis in frequency domain has been employed with the aim of mitigating three kinds of fluctuations, i.e. intra-day, intrahour and real time. Authors in [6] have had the objective of limiting 10-minute and 30-minute fluctuations of a wind farm by adding an optimal amount of ESS by means of an adaptive washout filter dispatch strategy. Brekken et al. have demonstrated simple rule-based and fuzzy dispatch controllers and compared the optimal amount of required ESS in each case, in order to achieve $\pm 4 \%$ of hour-ahead forecast in $90 \%$ of times [7]. In [8], the Area Control Error (ACE) parameter has been minimized using signal processing techniques like Fourier and Wavelet transforms.

In the present paper, a novel perspective to the problem of sizing of BESS for WF integration is introduced, in which the impact of WF layout on the required BESS capacity is investigated. Wind farm layout affects not only the total harvested energy but also the required capacity of BESS. Since both harvested energy level and BESS capacity directly influence the revenue, the effect of WF layout on these quantities are taken into account simultaneously and WF layout optimization problem is redefined.

Relative position of wind turbines (WT) according to speed and direction of wind flow directly affects the level of correlation of wind speed fluctuation in each turbines location [9]. The higher the correlation of wind fluctuation between pairs of turbines, the bigger capacity of BESS is needed to mitigate the impact of power fluctuations. A simple illustration of this phenomenon is given in Fig. 1, where the steep rise of the aggregated power output of a row of turbines placed perpendicular to wind flow can be compared to the more smooth and successive changes of the one with parallel positioning. It is obvious that the latter case requires lower BESS capacity to alleviate the output power fluctuations. Meanwhile, the wake effect which decreases the power production of downstream turbines compromises the required capacity of BESS. The effect of WF layout on power fluctuations has been examined in [10], but the authors have only investigated a snapshot of wind speed fluctuation over a specific time period and applied it to three different layouts, hence their approach does not take the stochastic property of wind into account. Furthermore, loss of production in WF due to the wake effect is not considered. Other researchers in the area

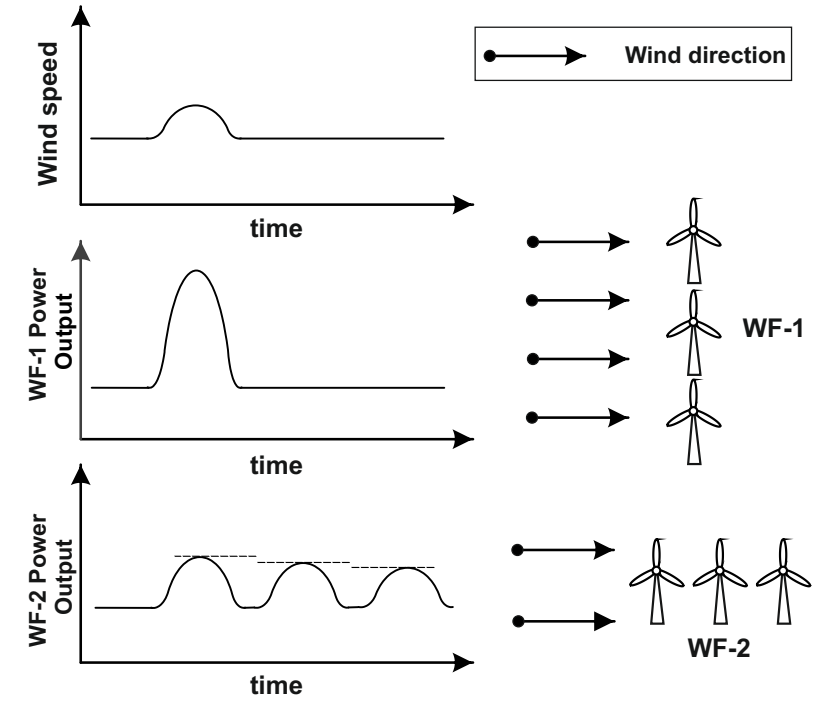

Fig. 1. The smoothing effect of different WF layouts.

of WF layout design and optimization have not discussed the effect of relative position of WTs on power fluctuations [11] [17].

This paper proposes a method to quantify the aforementioned correlation and its effect on the optimal capacity of BESS while considering the stochastic behavior of wind and resulting power fluctuation of wind farm. Wind speed variation in different turbine locations are treated as correlated stochastic processes with known power spectral density. Historical data of wind speed and direction are examined in a probabilistic manner. The method for determination of BESS power and energy capacity is taken from [7], which expresses total aggregated output of WF and ESS should be in $\pm 4 \%$ of hourahead forecast in $90 \%$ of the times. Persistence method is employed in this work for wind speed forecast. The proposed scheme has been tested by real wind data from Kentish Flats offshore wind farm in the UK in order to find an optimum layout.

The rest of the paper is organized as follows. Section II describes the methodology of the optimal sizing of the BESS for a specific WF layout. Section III is devoted to the objective function definition based on BESS capacity and annual harvested energy of the WF. The analytic formulation of the WF layout is also introduced and Genetic Algorithm is presented as the optimization method. Simulation of case studies in different scenarios is carried out in Section IV in order to evaluate the performance of the proposed method. Section V concludes the paper.

\section{Methodology of Sizing the BESS}

In this section, the Power Spectrum Density (PSD) theory is briefly described. Then, the methods of simulation of wind speed time series and calculation of BESS capacity are described. At the end, BESS optimum capacity for a specific layout is determined by means of historical data analysis. 


\section{A. Power Spectrum Density for a Stochastic Process}

If $x(t)$ is considered as a stochastic time-domain signal, the following time average represents its power:

$$
P=\lim _{T \rightarrow \infty} \frac{1}{2 T} \int_{-T}^{T} x(t)^{2} d t
$$

A truncated version of the signal over time period of $\mathrm{T}$ can be defined as:

$$
x_{T}(t)= \begin{cases}x(t) & |t| \leq T \\ 0 & |t|>T\end{cases}
$$

By integrating the truncated signal, Truncated Fourier Transform (TFT) is calculated and the frequency content of the signal can be analyzed over a finite time interval:

$$
X_{T}(f)=\int_{-\infty}^{+\infty} x_{T}(t)^{-j 2 \pi f t} d t=\int_{-T}^{T} x_{T}(t)^{-j 2 \pi f t} d t
$$

The PSD of the signal is then defined as [18]

$$
S_{x x}(f)=\lim _{T \rightarrow \infty} \frac{1}{2 T} E\left(\left|X_{T}(f)\right|^{2}\right)
$$

where $S_{x x}$ is the ensemble average of the TFT results. The distribution of power of the signal in different frequencies is described by the PSD given by (4).

\section{B. Simulation of Wind Speed Time Series}

Wind speed can be treated as a stochastic variable. Suppose vector $\mathbf{v}(t)=\left[v_{1}(t), v_{2}(t), \ldots, v_{N t}(t)\right]$ represents wind speed time series at each turbines hub height, in which every element is a stationary stochastic variable. The $N_{t} \times N_{t}$ Cross PSD (CPSD) matrix $S(f)$ for vector $v(t)$ is defined as:

$$
\mathbf{S}(\mathrm{f})=\left[\begin{array}{ccc}
\mathrm{S}_{11}(\mathrm{f}) & \cdots & \mathrm{S}_{1 N_{t}}(\mathrm{f}) \\
\vdots & \ddots & \vdots \\
\mathrm{S}_{N_{t} 1}(\mathrm{f}) & \cdots & \mathrm{S}_{N_{t} N_{t}}(\mathrm{f})
\end{array}\right]
$$

Diagonal elements are PSDs of wind speed in fixed turbine positions. By using curve fitting methods, analytical equations for PSD function in frequency domain can be obtained as explained in [19]. Equation (6) represents such a relationship:

$$
S_{i i}(f)=S_{H}(f)+S_{L}(f)
$$

Various expressions for $S_{H}(f)$ and $S_{L}(f)$ can be found in the literature and in the IEC standards [19], [20].

Here the concern is about fluctuations in the time scale of several seconds to a couple of hours, hence the proposed function in [20] for $S_{H}(f)$ is employed, which is valid for frequencies between $\frac{1}{0.02} s^{-1}$ to $\frac{1}{600} s^{-1}(50 \mathrm{~Hz}$ to $1.67 \mathrm{mHz}$ ):

$$
\begin{aligned}
& S_{H}(f)=\sigma_{w}^{2} \frac{2 \frac{L_{1}}{V_{w 0}}}{\left(1+6 \frac{L_{1}}{V_{w 0}} f\right)^{5 / 3}} \\
& L_{1}=\left\{\begin{array}{r}
5.67 z, \quad z<60 \mathrm{~m} \\
340.2 \mathrm{~m}, \quad z \geq 60 \mathrm{~m}
\end{array}\right.
\end{aligned}
$$

In order to include the frequencies below $\frac{1}{600} s^{-1}$, Sorensen [21] proposed an expression for PSD of wind fluctuation at

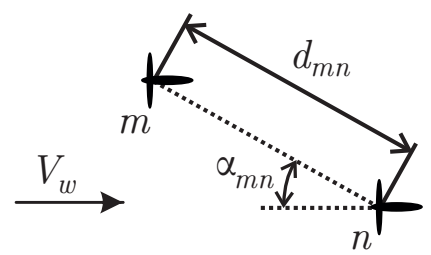

Fig. 2. Definition of inflow angle between turbines $m$ and $n$.

low frequencies:

$$
S_{L}(f)=\left(\alpha_{L} \cdot V_{w 0}+\beta_{L}\right)^{2} \frac{\frac{z}{V_{w 0}}}{\left(\frac{z . f}{V_{w 0}}\right)^{5 / 3}\left(1+100 \frac{z . f}{V_{w 0}}\right)}
$$

where $\alpha_{L}=0.0046$ and $\beta_{L}=0$ are based on the field measurements [22].

Off-diagonal elements are CPSD functions between each pair of $v_{i}(t)$ and $v_{j}(t), i, j=1,2,, N_{t}, i \neq j$ and can be determined by:

$$
S_{i j}(f)=\gamma_{i j}(f) \sqrt{S_{i i}(f) S_{j j}(f)}
$$

where $\gamma_{i j}(f)$ depends on both the distance between wind turbines and the inflow angle of wind. Corresponding to the CPSD matrix, the coherence matrix can be given by:

$$
\boldsymbol{\Gamma}(\mathrm{f})=\left[\begin{array}{cccc}
1 & \gamma_{12}(\mathrm{f}) & \cdots & \gamma_{1 N_{t}}(\mathrm{f}) \\
\gamma_{21}(\mathrm{f}) & 1 & \cdots & \gamma_{2 N_{t}}(\mathrm{f}) \\
\vdots & \vdots & \ddots & \vdots \\
\gamma_{N_{t} 1}(\mathrm{f}) & \gamma_{N_{t}}(\mathrm{f}) & \cdots & 1
\end{array}\right]
$$

A Davenport-type [23] coherence function is chosen to be implemented, and the decay factor is taken from [24]:

$$
\gamma_{m n}(f)=\mathrm{e}^{-a_{m n} \frac{d_{m n}}{V_{w 0}} f} \times \mathrm{e}^{-\mathrm{j} 2 \pi f \tau_{m n}}
$$

where $a_{m n}$ depends on the inflow angle $\alpha_{m n}$ as shown in Fig. 2. The second term in $\gamma_{m n}(f)$ definition corresponds to the time delay for the wave to traverse from turbine $m$ to turbine $n$, which takes $\tau_{m n}$ seconds. The decay factor suggested in [23] can be shown by the following expression:

$$
a_{m n}=\sqrt{\left(A_{\text {long }} \cos \left(\alpha_{m n}\right)\right)^{2}+\left(A_{\text {lat }} \sin \left(\alpha_{m n}\right)\right)^{2}}
$$

In order to include the smoothing effect on wind speed due to weighted averaging over the rotor of wind turbine, the equivalent PSD of wind speed at $i$ th turbine location is represented by:

$$
S_{i i, e q}(f)=F_{w t, i i}(f) \times S_{i i}(f)
$$

A numerical solution for $F_{w t, i i}(f)$ that gives an analytical expression is presented in [22], which depends on WT parameters as well as mean wind speed.

By using $S_{(i i, e q)}(f)$ instead of $S_{i i}(f)$ in (10), an equivalent CPSD matrix can be obtained denoted by $S_{e q}(f)$.

The mathematical method of generating random realizations of the stochastic wind speed time series $v(t)$ from the known $S_{e q}(f)$ and (f) in frequency domain, is based on [19] which is originally taken from [25].

The important point about $S_{e q}(f)$ and $\Gamma(f)$ matrices is that they are dependent on the mean wind speed, and $\Gamma(f)$ also depends on the wind direction and location of turbines in the farm. 


\section{Calculating Required Capacity of the ESS}

At a first step, the wind speed time series at turbine locations should be converted and aggregated to yield wind farm total power output. For the purpose of conversion of wind fluctuations to power fluctuations, a steady-state model of wind turbine is applied, taken from power curve of wind turbine. This assumption is reasonable because in this study the focus is on power fluctuations in the range of 1 minute to a couple of hours. Having power output of individual turbines, total power output of the wind farm can be found:

$$
P_{W F}=\sum_{i=1}^{N_{t}} P_{t_{i}}
$$

And aggregated power output of integrated WF and BESS would be:

$$
P_{O}=P_{b}+P_{W F}
$$

Next, the forecasted value of power output (i.e., the power the utility operator is expecting from the WF and BESS) should be determined. In this research, persistence method is used meaning the prediction of WF production of every hour is equal to the mean production of the previous hour. Because $T_{p e r}$ is set to 2 hours in this study, mean value of $P_{W F}$ in one hour is considered as the forecasted value for the other hour.

$P_{b}^{r}$ is defined as the minimum power rating of battery which keeps $P_{O}$ in the desired $\pm 4 \%$ band of the hour-ahead forecast. In order to calculate $P_{b, o p t}$ value, $P_{b}$ is defined as:

$$
P_{b}= \begin{cases}P_{f}-P_{W F}-0.04 & P_{f}-P_{W F}>0.04 \\ P_{f}-P_{W F}+0.04 & P_{f}-P_{W F}<-0.04 \\ 0 & \left|P_{f}-P_{W F}\right| \leq 0.04\end{cases}
$$

$P_{b}^{r}$ would be the maximum amplitude of $P_{b}$ in the time period of simulation:

$$
P_{b}^{r}=\max \left\{\left|P_{b}(t)\right|\right\} \quad 0<t \leq T_{\text {per }}
$$

The energy capacity of ESS is calculated from the following equations:

$$
\begin{gathered}
\eta=\left\{\begin{array}{cc}
\eta_{c} & P_{b}>0 \\
1 / \eta_{d} & P_{b}<0
\end{array}\right. \\
\Delta E_{b}=\eta \int_{0}^{T_{p e r}} P_{b} d t \\
E_{b}^{r}=\max \left(\Delta E_{b}\right)-\min \left(\Delta E_{b}\right)
\end{gathered}
$$

where for the sake of simplicity, it is assumed that the State of Charge (SoC) of battery can vary between zero and 1 .

$$
0 \leq S o C \leq 1
$$

\section{Historical Data Analysis and Determining ESS Optimum Capacity for a Specific Layout}

Two-dimensional Probability Density Function (PDF) of historical wind speed and direction data for the specific site of WF is generated. To obtain this PDF, the range of wind speed (from zero to maximum speed $-V_{\max }$ ) is divided into $N_{s}$ equal intervals, and 360 degrees direction is divided into $N_{d}$ equal intervals and all data are distributed in the corresponding bins.
Each bin is specified by wind speed $V_{i}$ and wind direction $d_{j}$ and can be shown by a pair of $\left(V_{i}, d_{j}\right)$. All $N_{s} \times N_{d}$ bins can be represented by the below wind speeds and directions:

$$
\begin{aligned}
V_{i} & =\frac{V_{\max }}{2 N_{s}}+(i-1) \cdot \frac{V_{\max }}{N_{s}} \quad ; \quad i=1,2, \ldots, N_{s} \\
d_{j} & =\frac{360^{\circ}}{2 N_{d}}+(j-1) \cdot \frac{360^{\circ}}{N_{d}} \quad ; \quad j=1,2, \ldots, N_{d}
\end{aligned}
$$

$P r_{i j}$ can be found by dividing the number of data in the $\left(V_{i}, d_{j}\right)$ bin denoted by $N_{i j}$, by the total number of available data points:

$$
P r_{i j}=\frac{N_{i j}}{\sum N_{i j}}
$$

For each pair of $\left(V_{i}, d_{j}\right)$, WF power time series is simulated repeatedly in order to generate a set of time series. The number of repetition is proportional to $P r_{i j}$. First, $P_{b}^{r}$ is determined for each realization of WF power time series according to the method described in Section II-C. Then, optimum battery capacity can be statistically quantified: take the resulting values of $P_{b}^{r}$ and compute its Cumulative Density Function (CDF). From the CDF of $P_{b}^{r}$, the rated power capacity of battery can be chosen to be the minimum value of which corresponds to the CDF equals or greater than 0.9 , so the BESS can meet power fluctuation requirements for $90 \%$ of the time.

$$
C D F\left(P_{b}^{r}=P_{b, o p t}\right) \geq 0.9
$$

After determining the $P_{b, o p t}$, the same procedure is performed to find $E_{b, o p t}$. $E_{b}^{r}$ values are calculated according to (20), with an exception that the value of $P_{b}$ is restricted to $P_{b, o p t}$, determined previously. $E_{b, o p t}$ would be the maximum value in the set of the entire $E_{b}^{r}$ values.

It is worth mentioning that as a consequence of applying stochastic method for simulation of the time series, each realization of WF power time series leads to different values for $P_{b}^{r}$ and $E_{b}^{r}$. Thus, the resulting $P_{b, o p t}$ and $E_{b, o p t}$ vary in each simulation.

\section{OBjective FunCtion Definition AND OPTIMIZATION}

\section{A. Calculating Average Annual Production of the WF Con- sidering Wake Effect}

Due to wake effect, the average wind speed drops behind the rotor of wind turbines and the turbulent wind passes through downstream wind turbines. Hence, the wake effect is the major cause of reduction in power production of wind farms.

Different wake effect modeling approaches have been addressed in the literature [26]-[28]. Based on its relative simplicity [27], [28] and reasonable results for power system studies [29], the current study is conducted on the basis of Jensen model [26]. The method of calculation of wake effect is described in Appendix A.

Considering the PDF of wind speed and direction, mean annual harvested power (corresponding to the capacity factor of the WF) can be determined by the following formula:

$$
\bar{P}_{\text {annual }}=\sum_{i, j} \operatorname{Pr}_{i j} \times P_{W F, a v}\left(V_{i}, d_{j}\right)
$$


where $P_{W F, a v}\left(V_{i}, d_{j}\right)$ denotes steady state WF power production with mean wind speed $V_{i}$ and with direction $d_{j}$, taking wake effect into account.

\section{B. Objective Function Definition and Optimization of WF Layout}

The WT arrays in most large, existing offshore WFs are arranged in rectilinear grids (e.g. Nysted, Egmond aan Zee and Kentish Flats). This simple approach is based on a rule of thumb: the distance between turbines in the prevailing wind direction is between 5 to 9 rotor diameters, and 3 to 5 in the crosswind direction [30]. Despite its simplicity, this method may miss the opportunity of better energy yield of the WF. Extensive research has been conducted to date on the WF layout optimization. In previous studies, various heuristic optimization techniques have been applied, including Genetic Algorithm (GA) [11], [12], Particle Swarm Optimization (PSO) [14], [17], Simulated Annealing (SA) [13] and Random Search (RS) [15]. In [30] a comparative study of these methods has been conducted. Sequential Convex Programming (SCP) has been used in [16] which outperforms GA in terms of finding the optimum solution.

The objective function is defined from the viewpoint of the wind farm owner in order to maximize its benefit. The benefit of wind farm owner consists of two parts, total revenue of electricity production during the WF lifetime, and investment costs. Here only the investment cost of BESS is taken into account since other investment costs are irrelevant to wind farm layout ${ }^{1}$. Based on [7], the cost model for flow battery energy storage devices is defined as:

$$
C_{B E S S}=\mathrm{c}_{P} \times P_{b, o p t}+\mathrm{c}_{E} \times E_{b, o p t}
$$

where $c_{P}$ mainly depends on the power conversion system, while $c_{E}$ is based on BESS technology. Empirical values of $600 \$ / \mathrm{kW}$ and $650 \$ / \mathrm{kWh}$ are assumed for $c_{P}$ and $c_{E}$, respectively [31], [32]. The value of $c_{E}$ is based on Lithiumion batteries which is taken from Table I, where four major BESS technologies and their respective parameters are listed [31], [32]. Lithium-ion technology exhibits high efficiency while having a reasonable price per $\mathrm{kWh}$.

TABLE I

PARAMETERS OF DIFFERENT BESS TECHNOLOGIES

\begin{tabular}{lll}
\hline \hline Battery Technology & $c_{E}(\$ / k W h)$ & Efficiency $(\%)$ \\
\hline Vanadium redox (VR) & 700 & 70 \\
$\begin{array}{l}\text { Advanced lead-acid } \\
\text { (Pb-acid) }\end{array}$ & 1000 & 80 \\
Lithium-ion (Li-ion) & 650 & 90 \\
Sodium Sulphur (NaS) & 450 & 85 \\
\hline \hline
\end{tabular}

The present value of the total revenue of wind farm during the battery lifetime period is calculated as follows:

$\mathrm{PV}\left(\operatorname{Rev}_{W F}\right)=\frac{(1+I)^{T_{B E S S}}-1}{I(1+I)^{T_{B E S S}}} \times \bar{P}_{\text {annual }} \times 8760 \times \rho_{W E}$

\footnotetext{
${ }^{1}$ Actually array cable length depends on WF layout, but for optimal layout turbines are placed as far as possible in a confined area due to wake effect. Thus comparing different layouts with maximum annual power production, there are small changes of array cable length that are negligible.
}

The first term accounts for converting the annual values to the present value [33], while the rest presents the annual revenue of selling energy to the grid considering 8760 hours of operation per year.

Optimization of the overall objective function is given as:

$$
\operatorname{Max}\left\{\mathrm{PV}\left(\operatorname{Rev}_{W F}\right)-C_{B E S S}\right\}
$$

where the BESS cost is deducted from the present value of the revenue in order to yield the net profit. Then the resulting function is maximized.

Based on each $\left(V_{i}, d_{j}\right)$ wind characteristics and for a given wind farm layout, objective function can be evaluated.

In general, WT locations in a WF can be shown by a set of $\mathrm{x}$ and $\mathrm{y}$ coordinates. Let $X=\left\{x_{1}, x_{2}, \ldots, x_{N_{t}}\right\}$ and $Y=$ $\left\{y_{1}, y_{2}, \ldots, y_{N_{t}}\right\}$ be sets of $x$ and $y$ coordinates of $N_{t}$ WTs in the plane. The goal is to find a set of coordinates such that the defined objective function is maximized. Furthermore, the layout has to satisfy certain constraints.

Two main restrictions for each turbine coordinates should be considered: placement inside the confined area of the wind farm, and the minimum distance between each pair of the turbines. The WF boundaries can be represented by linear $\mathrm{x}-\mathrm{y}$ equations according to the shape of the area. The minimum distance constraint can be expressed as:

$$
\begin{gathered}
\left(x_{i}-x_{j}\right)^{2}+\left(y_{i}-y_{j}\right)^{2} \geq d_{\text {min }}{ }^{2} \\
\forall i, j \in\left\{1,2, \ldots, N_{t}\right\}
\end{gathered}
$$

where $d_{\text {min }}$ is practically three to five times of the diameter of the WT rotor. In this paper, it is chosen to be four times of the rotor diameter.

Due to the fact that most offshore WFs are organized in rectilinear grids, the shape of WF layout is basically considered as a rectangle which is tilted and rotated. The coordinates inside a rectangular area can be expressed in the form of:

$$
\begin{gathered}
X_{\min }<x_{i}<X_{\max } ; Y_{\min }<y_{i}<Y_{\max } \\
\forall i, j \in\left\{1,2, \ldots, N_{t}\right\}
\end{gathered}
$$

And the tilt can be formulated as

$$
\begin{gathered}
x_{i}^{\prime}=x_{i}+\frac{\left(Y_{\max }-y_{i}\right)}{Y_{\max }} * X_{T} \\
\forall i, j \in\left\{1,2, \ldots, N_{t}\right\}
\end{gathered}
$$

where $x_{i}^{\prime}$ is the new $x$ coordinate after tilting. With $X_{T}$ value of zero, the resulting layout is rectangle and with increasing tilt factor it becomes more like a parallelogram. After tilting, the rotation around the origin is performed to create the final layout. Hence, the values of $X_{T}$ and rotation are considered as two decision variables.

There are two general approaches for solving this problem, continuous space and discrete. As $x$ and $y$ coordinates can have any real value inside the boundaries of the problem, our search space is continuous. Due to the complexity of WF layout optimization, Genetic Algorithm (GA) is employed to solve the problem in this work which is also widely used by many researchers in this field, e.g. [11], [12], [34]. Unlike the traditional calculus-based methods, GAs are global and do not require the derivatives of objective functions.

GA first generates a population of chromosomes randomly. 


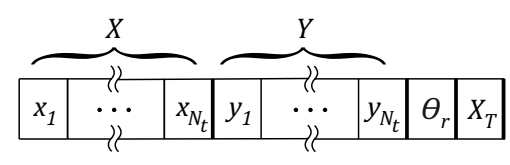

Fig. 3. Definition of chromosome in GA

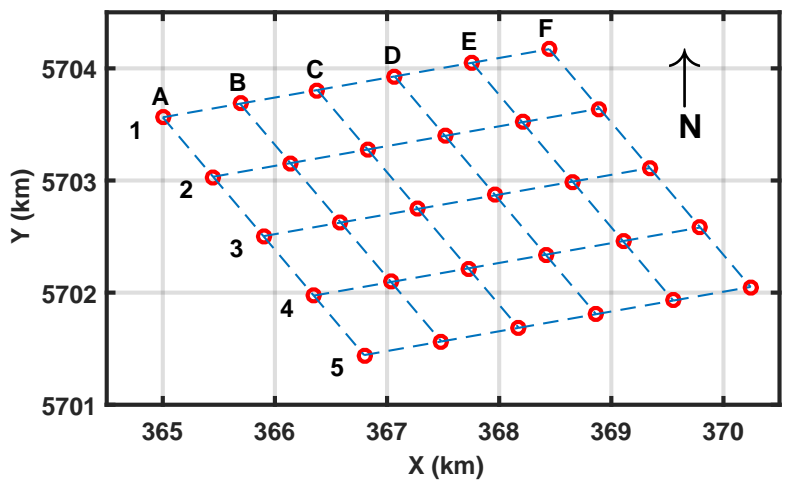

Fig. 4. Kentish Flats WF layout.

Every chromosome is an individual which represents a WF layout. The three major steps of GA after initialization are selection, crossover and mutation, which are not explained here for the sake of brevity. The algorithm repeats until the maximum number of generations is reached. More details about implementation of GA can be found in the literature, e.g. [35] and [36].

The definition of chromosome is illustrated in Fig. 3. Values of the $\mathrm{x}$ and $\mathrm{y}$ coordinates are put first in the chromosome, followed by rotation angle and tilt factor of the layout.

\section{Case Study and Discussion}

The stochastic simulation procedure and optimization method described in previous sections have been applied to Kentish Flats WF. It is located in South East coast of the UK and is in operation since 2005. It comprises 30 wind turbines rated at $3 \mathrm{MW}$ each, with rotor diameter of $90 \mathrm{~m}$. The details of WT parameters are given in Appendix B. The layout of Kentish Flats WF is shown in Fig. 4. The distance between the turbines in a column and in a row is $700 \mathrm{~m}$ corresponding to 7.8 rotor diameter.

Wind speed and direction data are gathered from a meteorological mast in WF location, averaged on 10-min intervals during 2010 [37] . The corresponding wind rose is depicted in Fig. 5. The number of bins for wind speed and direction are $N_{s}=20$ and $N_{d}=36$ in the simulation.

To show the performance of the introduced method in Section II, it is applied to the actual Kentish Flats WF layout first and the detailed results are presented. Then, two different scenarios are presented and the optimization results are discussed.

Fig. 6 illustrates the wind speed time series of three WTs in Kentish Flats WF for a period of 2 hours with mean wind speed of $11 \mathrm{~m} / \mathrm{s}$, wind direction of $260^{\circ}$ and turbulence intensity of $5 \%$. As Fig. 6 shows, the average wind speed at WT F2 location is the lowest due to wake effect. Furthermore, comparing wind speed at locations of WT A2 and WT F2 indicates a delay of about 5 minutes which corresponds very

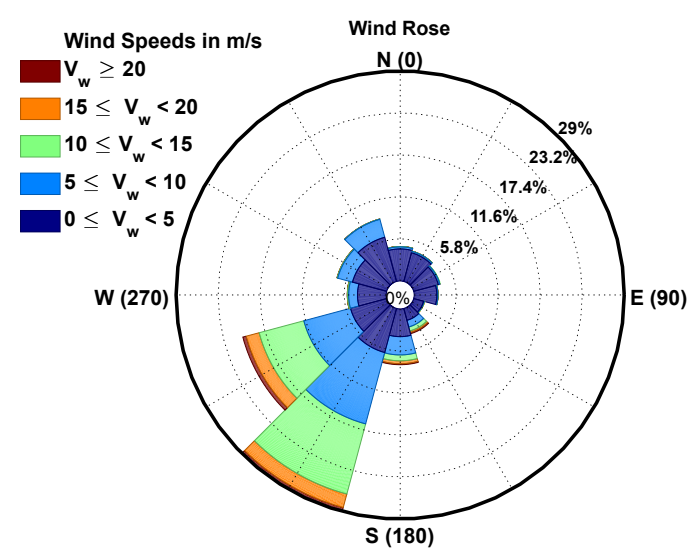

Fig. 5. Wind rose for Kentish Flats.

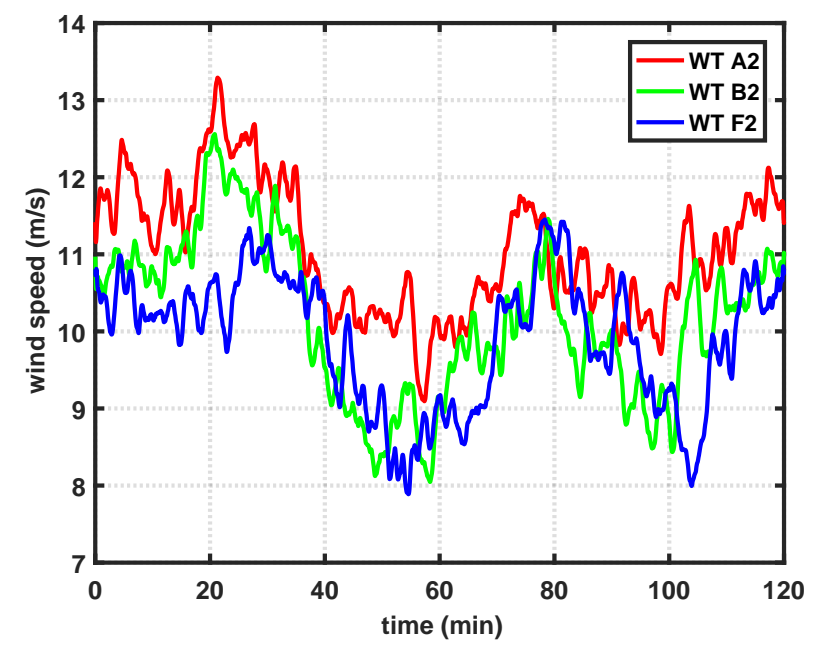

Fig. 6. Wind speed at different WT locations.

well to the expected delay for $11 \mathrm{~m} / \mathrm{s}$ with $5 \times 700 \mathrm{~m}$ distance.

Fig. 7 demonstrates how the battery parameters are calculated based on power output of the WF and the hourly forecasted values. The base power for the per unit values equals the installed capacity of the WF, which is $90 \mathrm{MW}$.

In order to assess the effectiveness of the proposed method in reduction of fluctuation of WF power output, the difference between the forecasted power and the actual output of the WF is defined as $d P$.

$$
d P=P_{o}-P_{f}
$$

The histogram of $d P$ is depicted in Fig. 8, which is calculated before integration of BESS into WF. Furthermore, considering power deviations greater than 0.04 p.u. are not acceptable, a Power Fluctuation Index (PFI) can be assigned to the WF power output, formulated as follows:

$$
P F I=\frac{\sum N_{x} \times\left|d P_{x}\right|}{\sum N_{x}} \times 100
$$

which sums the unacceptable power deviations $\left(d P_{x}\right)$ and normalizes the result.

Energy curtailment is the other important indicator that can demonstrate and quantify the power fluctuation of WF, which is previously employed in the area of wind energy studies [38]-[40]. If curtailment is required by the grid, the 


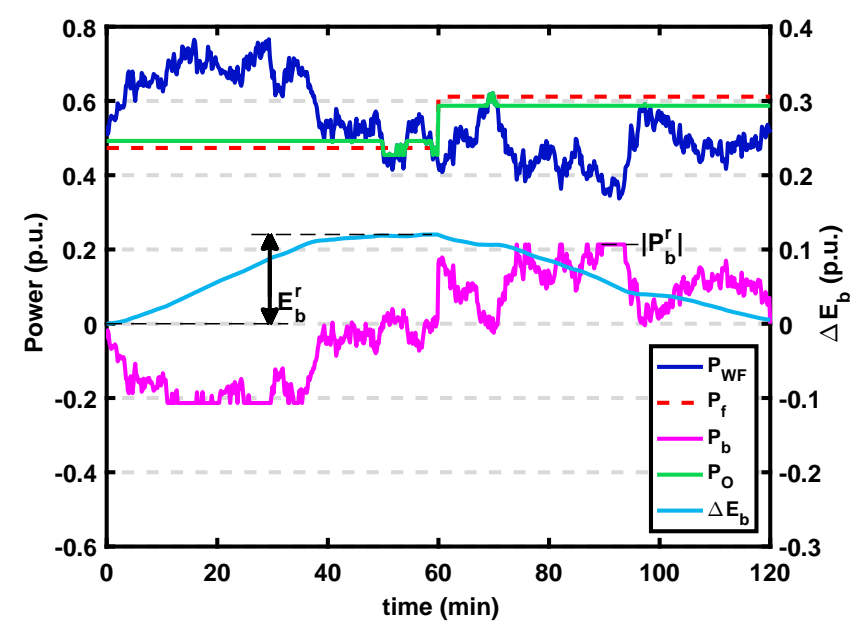

Fig. 7. WF and BESS output power and forecasted value.

output power of WF should be curtailed to meet the power setpoint. This condition occurs when $d P$ is greater than a certain threshold, which is set to 0.04 of the forecasted power in our study.

$$
\begin{aligned}
& d P_{\text {curt }}=\left\{\begin{array}{lr}
d P-0.04 \times P_{f} & d P>0.04 \times P_{f} \\
0 & \text { otherwise }
\end{array}\right. \\
& E_{\text {curt }}=\int P_{\text {curt }} \cdot d t
\end{aligned}
$$

Table II shows the required battery parameters, annual harvested energy and capacity factor for Kentish Flats WF based on 2010 data, as well as annual curtailed energy and PFI. Energy curtailment is calculated without considering BESS in order to demonstrate the impact of WF layout on power fluctuation comparing with the original layout. In objective function evaluation, the values of $\rho_{W E}, T_{B E S S}$ and I are 0.1 $\$ / k W h, 15$ years and 0.03 , respectively.

TABLE II

Results for the ACtual Layout of Kentish Flats WF

\begin{tabular}{ll}
\hline \hline$P_{b, o p t}(p . u)$. & 0.120 \\
$E_{b, o p t}(p . u)$. & 0.056 \\
Rotation of the layout (degree) & 9.0 \\
Tilt factor of the layout & 1400 \\
Annual harvested energy (GWh) & 251.3 \\
Capacity factor (\%) & 31.89 \\
Energy curtailment-without BESS (GWh/year) & 29.1 \\
PFI (\%) & 10.45 \\
Objective function value $(\$)$ & $290.4 \times 10^{6}$ \\
\hline \hline
\end{tabular}

The optimization method is performed on two scenarios: the first scenario considers constant wind speed and direction, and the second one uses real wind data. The GA parameters are shown in Table III. In order to generate initial population of the $\mathrm{GA}$, random deviations are added to the $\mathrm{x}$ and $\mathrm{y}$ coordinates of the WTs in the actual WF layout.

TABLE III

PARAMETERS OF Genetic Algorithm

\begin{tabular}{ll}
\hline \hline Population size & 50 \\
Selection rate & 0.85 \\
Mutation rate & 0.05 \\
Maximum number of generations & 200 \\
\hline \hline
\end{tabular}

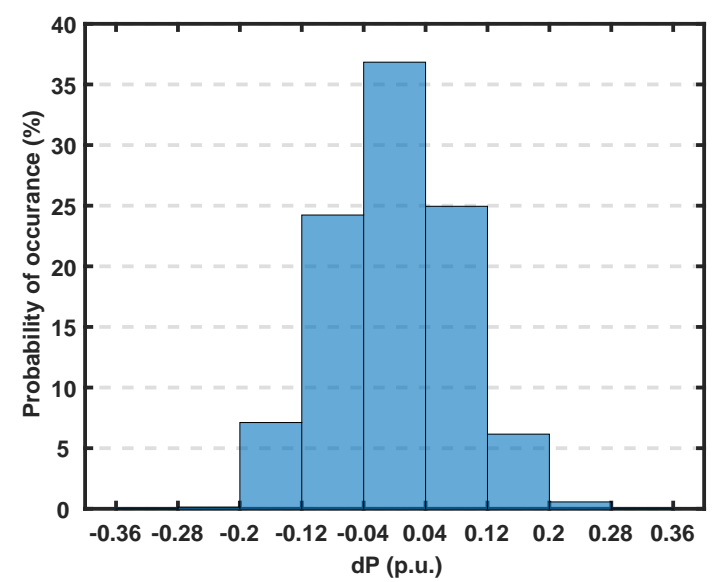

Fig. 8. Histogram of dP for WF in original layout..

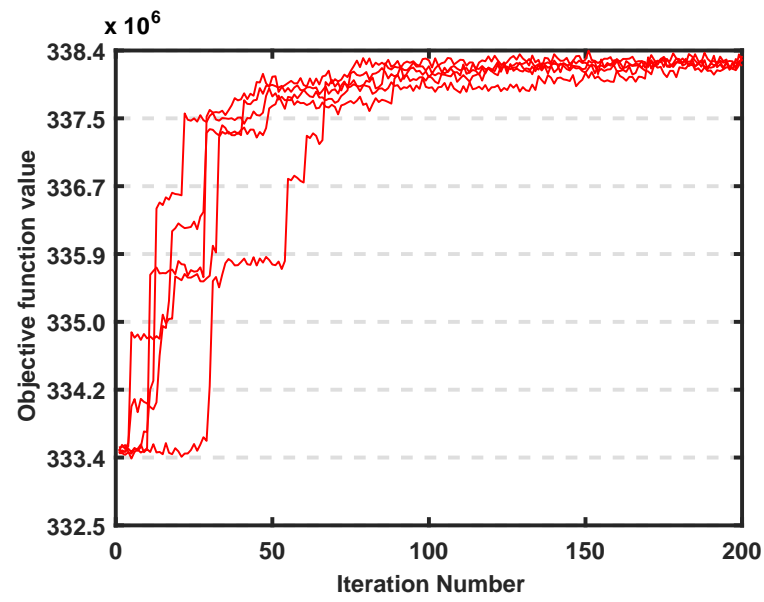

Fig. 9. Convergence curves of the objective function during 200 iterations.

\section{A. First Scenario: Constant Wind Speed and Direction}

Constant wind speed and direction are set to the long term average values in Kentish Flats site, which are $210^{\circ}$ and $8.2 \mathrm{~m} / \mathrm{s}$, respectively. The results are provided in Table IV. Comparing the capacity factors in Table IV in two conditions

TABLE IV

RESULTS FOR THE OPTIMUM LAYOUT-FIRST SCENARIO

\begin{tabular}{ll}
\hline \hline$P_{b, o p t}(p . u)$. & 0.161 \\
$E_{b, o p t}(p . u)$. & 0.106 \\
Rotation of the layout (degree) & 11.8 \\
Tilt factor of the layout & 1471 \\
Capacity factor-considering wake effect $(\%)$ & 37.52 \\
Capacity factor-without wake effect (\%) & 37.79 \\
Energy curtailment-without BESS (GWh/year) & 35.6 \\
PFI(\%) & 10.87 \\
Objective function value (\$) & $338.2 \times 10^{6}$ \\
\hline \hline
\end{tabular}

reveals that with constant wind direction, GA can optimize the layout in a way that minimum wake loss occurs in the farm, hence the maximum energy harvesting is guaranteed in this case. PFI and curtailed energy are increased comparing with the original layout.

Fig. 9 shows the iteration curve of the optimization process by GA, repeated five times. All the iteration curves reached the same optimal value, while different runs exhibit different evolution paths through the final value. It can be seen that GA 


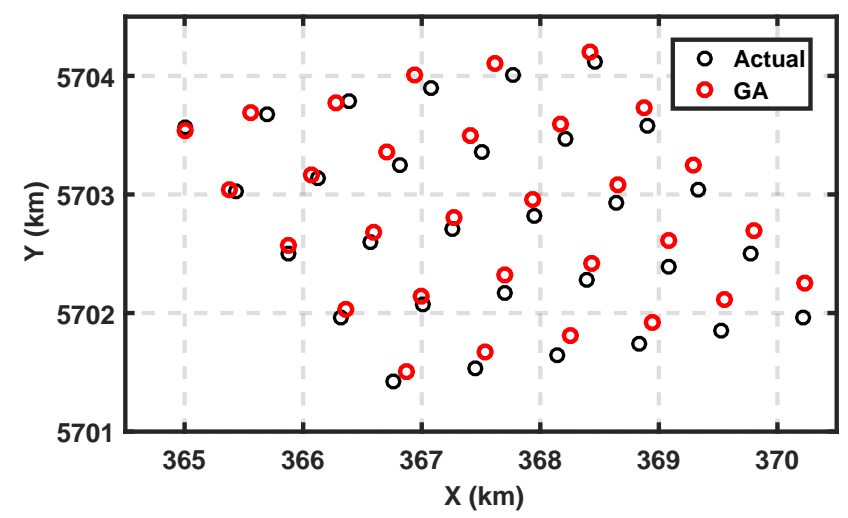

Fig. 10. Optimum layout in the first scenario.

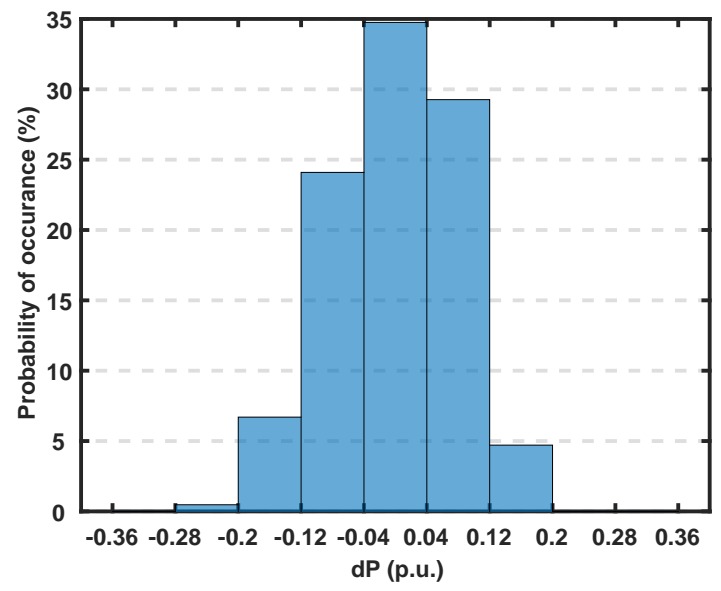

Fig. 11. Histogram of $\mathrm{dP}$ for $\mathrm{WF}$ in the first scenario.

finds the optimum layout almost around 150th generation. The spikes along the iteration curve is caused by the varying $P_{b, o p t}$ and $E_{b, o p t}$, which in turn results from the stochastic nature of the time series simulation method, as described earlier in Section II-D. The optimum layout in the first scenario is illustrated in Fig. 10. One can observe that the rotation and tilt values are increased comparing to that of the actual layout in accordance to the prevailing and constant wind direction.

Fig. 11 shows the histogram of output power deviation of the WF in absence of BESS, for the first scenario. Compared to the histogram of the original layout, it can be observed that the acceptable power deviations in the range of \pm 0.04 p.u. are decreased. In contrast, the increment of unacceptable power deviations is well aligned with the larger value of PFI compared to the original layout.

\section{B. Second Scenario: Real Wind Data}

In this scenario, real wind data from 2010 are utilized and the numerical results are provided in Table V.

The objective function value in this scenario is increased by $0.79 \%$ comparing with the actual WF results. However, the increase in capacity factor is only $0.50 \%$ which is smaller than that of objective function value. The reason lies in the fact that the actual layout has been optimized based on maximizing the capacity factor, but in the current optimization, the BESS capacity is also taken into account. Hence, the improvement in BESS capacity is more evident, i.e. $9.2 \%$ and $7.1 \%$ decrease
TABLE V

RESULTS FOR THE OPTIMUM LAYOUT-SECOND SCENARIO

\begin{tabular}{ll}
\hline \hline$P_{b, \text { opt }}($ p.u. $)$ & 0.109 \\
$E_{b, \text { opt }}($ p.u. $)$ & 0.052 \\
Rotation of the layout (degree) & 6.7 \\
Tilt factor of the layout & 1388 \\
Capacity factor (\%) & 32.05 \\
Energy curtailment-without BESS (GWh/year) & 33.4 \\
PFI(\%) & 10.03 \\
Objective function value (\$) & $292.7 \times 10^{6}$ \\
\hline
\end{tabular}

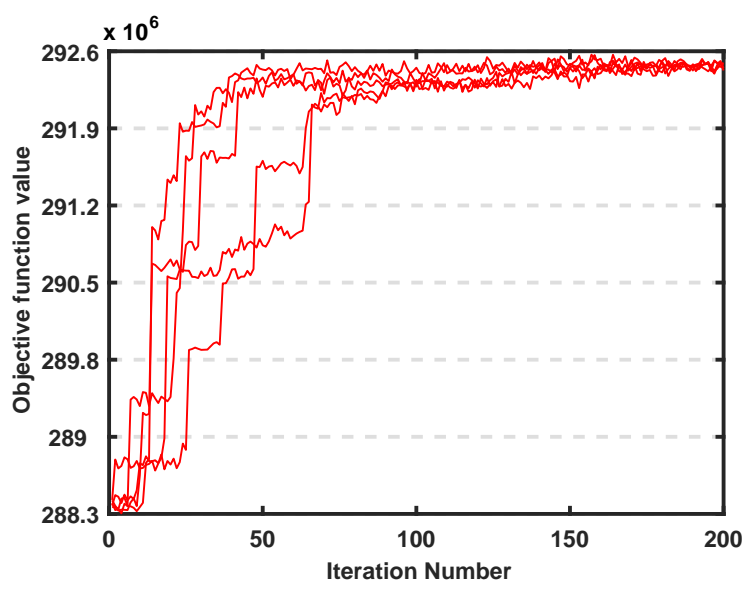

Fig. 12. Convergence curves of the objective function during 200 iterations.

for power and energy capacity of BESS, respectively. It is worth noting that the increment in objective function value of $\$ 2.2106$ can justify the implementation of a custom layout, taking into account the installation costs of about tens of million dollars for offshore WFs [41].

Fig. 12 shows the iteration curves of the optimization process by GA. The same as previous scenario, the optimal value of all five runs are the same. It can be seen that GA finds the optimum layout almost around 170th generation. The optimum layout is depicted in Fig. 13. In this scenario, the rotation and tilt factor are decreased compared to that of the actual layout.

The histogram of output power deviation in second scenario is illustrated in Fig. 14. Same as the first scenario, BESS is not considered in evaluation of histogram. Compared to the histogram of the original layout, the unacceptable deviations are decreased, which can be observed from the improved PFI value.

\section{BESS efficiency analysis}

Different BESS technologies have various efficiency values $(\eta)$, as shown in Table I. It is assumed that $\eta_{c}^{2}=\eta_{d}^{2}=\eta$ in (19). In order to assess the impact of different efficiencies on the outcome of the optimization process, objective function is evaluated for all presented BESS types. The results are presented in Table VI. It can be concluded that the impact of BESS efficiency on $E_{b, o p t}$ value is more evident than the other parameters. The other important factor is $c_{E}$ which causes the objective function value to differ among the four cases. For advanced lead-acid battery which has the largest $c_{E}$, the objective function value is even degraded comparing with the original layout. In contrary, NaS battery which has the lowest 


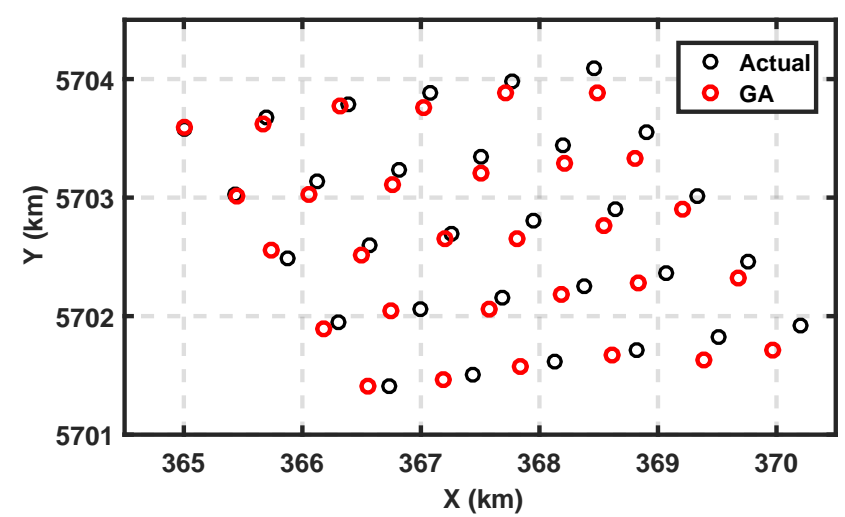

Fig. 13. Optimum layout in the second scenario.

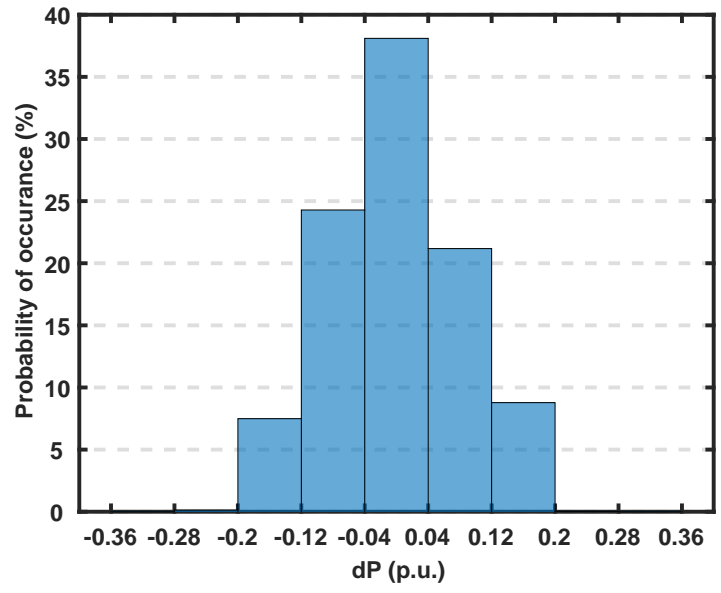

Fig. 14. Histogram of dP for WF in the second scenario.

TABLE VI

Objective Function Evaluation Considering DifFerent BESS TECHNOLOGIES

\begin{tabular}{llll}
\hline \hline BESS Technology & $P_{b, o p t}(p . u)$. & $E_{b, o p t}(p . u)$. & $\begin{array}{l}\text { Objective Function } \\
\text { Value }\left(\times 10^{6} \$\right)\end{array}$ \\
\hline Vanadium redox & 0.110 & 0.058 & 291.6 \\
Advanced lead-acid & 0.111 & 0.055 & 290.2 \\
Lithium-ion & 0.109 & 0.052 & 292.7 \\
Sodium Sulphur & 0.109 & 0.053 & 293.6 \\
\hline \hline
\end{tabular}

$c_{E}$ performs better than Li-ion battery in terms of objective function value, while Li-ion has slightly better efficiency.

Fig. 15 depicts the optimum layouts with different BESS technologies. The optimum layouts have slight differences, and one can observe that the case with advanced lead-acid battery which is the most expensive alternative and has the lowest efficiency, is more similar to the original layout.

\section{CONCLUSION}

In contrast to the conventional methods of WF layout optimization which maximize the energy production, this paper proposed a novel method to optimize the energy production and the required capacity of BESS in a WF, simultaneously. Based on the PSD theory, it has been shown that the layout affects the level of output fluctuation of a WF. The level of fluctuation of a WF directly influence the power and energy capacities of the required BESS. Thus, the joint optimization has been realized by defining an objective function including both the BESS cost and the revenue from the sale of wind

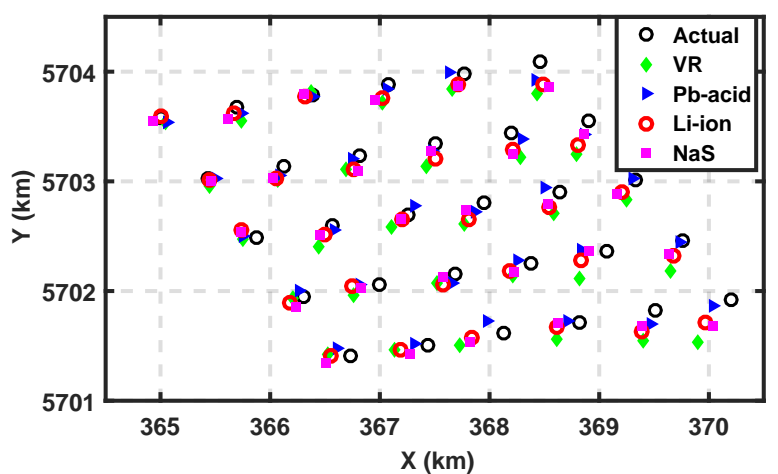

Fig. 15. Optimum layouts with different BESS technologies.

energy. The GA-based optimization method performed well on the real data of Kentish Flats WF in terms of reduction of power fluctuation which has been quantified by the introduced Power Fluctuation Index (PFI). Furthermore, unacceptable power deviations have been reduced in the optimum layout, leading to less energy curtailment. Two different scenarios have been analyzed and the results have been compared with the actual WF layout, suggesting that the proper design of WF leads to lower power fluctuation and hence less required BESS capacity. Various BESS technologies with different efficiency and cost parameters have been studied, and the sensitivity analysis suggested a compromise between storage cost and efficiency. In future studies, advanced BESS control schemes can be employed to further optimize the BESS capacity. In addition, operation and maintenance costs can be taken into account in objective function definition.

\section{APPENDIX A}

In Jensen model for the wake effect, it is assumed that the wake expands linearly behind a WT. The velocity deficit inside the wake area $(u)$ is defined as the fractional reduction of the free wind speed, which in distance $x$ from the turbine is computed as:

$$
u=\frac{1-\sqrt{1-C_{t}}}{(1+(2 k x / D))^{2}}
$$

where $C_{t}, k$ and $D$ are the thrust coefficient, decay factor and rotor diameter, respectively. In the current study, a decay factor of 0.075 is chosen, according to [22].

When the turbine $n$ is influenced by the wakes of multiple turbines, the aggregated velocity deficit $u_{n}$ is calculated as:

$$
u_{n}=\sqrt{\sum u^{2}}
$$

Partial wake occurs when one turbine cuts the wake area of another turbine. Detailed discussion about formulation of the partial wake can be found in [29]. Taking wake losses into account, $P_{W F, a v}$ in (26) is formulated as follows:

$$
P_{W F, a v}\left(V_{i}, d_{j}\right)=\sum_{n=1}^{N_{t}} P_{t_{n}}\left(V_{n}\right) ; V_{n}=V_{i} .\left(1-u_{n}\right)
$$

\section{APPENDIX B}

\section{ACKNOWLEDGMENT}

The main part of this work is conducted at Imperial College London during a six-month graduate student exchange pro- 
TABLE VII

PARAMETERS OF THE WIND TURBINE

\begin{tabular}{ll}
\hline \hline Nominal power (MW) & 3.0 \\
Hub height $(\mathrm{m})$ & 70 \\
Rotor diameter $(\mathrm{m})$ & 90 \\
Thrust coefficient $-C_{T}$ & 0.82 \\
Rated speed $(\mathrm{m} / \mathrm{s})$ & 15 \\
Cut-in speed $(\mathrm{m} / \mathrm{s})$ & 4 \\
Cut-off speed $(\mathrm{m} / \mathrm{s})$ & 25 \\
\hline \hline
\end{tabular}

gram. The authors gratefully acknowledge support from Sharif University of Technology and Imperial College London.

\section{REFERENCES}

[1] Q. Li, S. S. Choi, Y. Yuan, and D. Yao, "On the determination of battery energy storage capacity and short-term power dispatch of a wind farm," IEEE Transactions on Sustainable Energy, vol. 2, no. 2, pp. 148-158, 2011.

[2] T. Ackermann, Wind Power in Power Systems. Wiley, 2012.

[3] C. Luo and B.-T. Ooi, "Frequency deviation of thermal power plants due to wind farms," IEEE Transactions on Energy Conversion, vol. 21, no. 3, pp. 708-716, 2006.

[4] M. Black and G. Strbac, "Value of bulk energy storage for managing wind power fluctuations," IEEE Transactions on Energy Conversion, vol. 22, no. 1, pp. 197-205, 2007.

[5] Q. Jiang, Y. Gong, and H. Wang, "A battery energy storage system dual-layer control strategy for mitigating wind farm fluctuations," IEEE Transactions on power systems, vol. 28, no. 3, pp. 3263-3273, 2013.

[6] Y. V. Makarov, P. Du, M. C. Kintner-Meyer, C. Jin, and H. F. Illian, "Sizing energy storage to accommodate high penetration of variable energy resources," IEEE Transactions on sustainable Energy, vol. 3, no. 1, pp. 34-40, 2012.

[7] T. K. Brekken, A. Yokochi, A. Von Jouanne, Z. Z. Yen, H. M. Hapke, and D. A. Halamay, "Optimal energy storage sizing and control for wind power applications," IEEE Transactions on Sustainable Energy, vol. 2, no. 1, pp. 69-77, 2011.

[8] H. Bitaraf, S. Rahman, and M. Pipattanasomporn, "Sizing energy storage to mitigate wind power forecast error impacts by signal processing techniques," IEEE Transactions on Sustainable Energy, vol. 6, no. 4, pp. 1457-1465, 2015.

[9] P. S. Veers, "Three-dimensional wind simulation," Sandia National Labs., Albuquerque, NM (USA), Tech. Rep., 1988.

[10] A. Johnson, A. Escobar, J. Balda, and A. Barnes, "Wind farm layout for mitigating output power intermittency," in Power Electronics for Distributed Generation Systems (PEDG), 2012 3rd IEEE International Symposium on. IEEE, 2012, pp. 883-889.

[11] H. Long and Z. Zhang, "A two-echelon wind farm layout planning model," IEEE Transactions on Sustainable Energy, vol. 6, no. 3, pp. 863-871, 2015.

[12] S. Grady, M. Hussaini, and M. M. Abdullah, "Placement of wind turbines using genetic algorithms," Renewable energy, vol. 30, no. 2, pp. $259-270,2005$

[13] R. A. Rivas, J. Clausen, K. S. Hansen, and L. E. Jensen, "Solving the turbine positioning problem for large offshore wind farms by simulated annealing," Wind Engineering, vol. 33, no. 3, pp. 287-297, 2009.

[14] H. Yang, K. Xie, H.-M. Tai, and Y. Chai, "Wind farm layout optimization and its application to power system reliability analysis," IEEE Transactions on Power Systems, vol. 31, no. 3, pp. 2135-2143, 2016.

[15] J. Feng and W. Z. Shen, "Solving the wind farm layout optimization problem using random search algorithm," Renewable Energy, vol. 78, pp. 182-192, 2015

[16] J. Park and K. H. Law, "Layout optimization for maximizing wind farm power production using sequential convex programming," Applied Energy, vol. 151, pp. 320-334, 2015.

[17] P. Hou, W. Hu, C. Chen, M. Soltani, and Z. Chen, "Optimization of offshore wind farm layout in restricted zones," Energy, vol. 113, pp. 487-496, 2016.

[18] S. Millers and D. Childers, Probability and random processes. Academic Press, 2012.

[19] P. Sørensen, A. D. Hansen, and P. A. C. Rosas, "Wind models for simulation of power fluctuations from wind farms," Journal of wind engineering and industrial aerodynamics, vol. 90, no. 12, pp. 13811402,2002
[20] “Wind Turbines-Part 1: Design requirements," Int. Std. IEC 61400-1, 3rd Ed., 2005-2008.

[21] P. Sørensen, N. A. Cutululis, A. Vigueras-Rodríguez, L. E. Jensen, J. Hjerrild, M. H. Donovan, and H. Madsen, "Power fluctuations from large wind farms," IEEE Transactions on Power Systems, vol. 22, no. 3, pp. 958-965, 2007.

[22] P. E. Sørensen, P. Pinson, N. A. Cutululis, H. Madsen, L. E. Jensen, J. Hjerrild, M. Heyman Donovan, J. Runge Kristoffersen, and A. Vigueras-Rodriguez, "Power fluctuations from large wind farms-final report," Ris $\varnothing$ DTU, Roskilde, Denmark, Tech. Rep. Ris $\varnothing-\mathrm{R}-1765,2009$.

[23] A. G. Davenport, "The spectrum of horizontal gustiness near the ground in high winds," Quarterly Journal of the Royal Meteorological Society, vol. 87, no. 372, pp. 194-211, 1961.

[24] W. Schlez and D. Infield, "Horizontal, two point coherence for separations greater than the measurement height," Boundary-Layer Meteorology, vol. 87, no. 3, pp. 459-480, 1998.

[25] M. Shinozuka and C.-M. Jan, "Digital simulation of random processes and its applications," Journal of sound and vibration, vol. 25, no. 1, pp. $111-128,1972$

[26] N. O. Jensen, "A note on wind generator interaction," Risø National Laboratory, Tech. Rep. Risø-M-2411, 1983. [Online]. Available: http://www.risoe.dtu.dk/rispubl/vea/veapdf/ris-m-2411.pdf

[27] K. Thomsen and H. A. Madsen, "A new simulation method for turbines in wakeapplied to extreme response during operation," Wind Energy, vol. 8 , no. 1 , pp. $35-47,2005$

[28] J. F. Ainslie, "Calculating the flowfield in the wake of wind turbines," Journal of Wind Engineering and Industrial Aerodynamics, vol. 27, no. 1-3, pp. 213-224, 1988.

[29] S. Kuenzel, L. P. Kunjumuhammed, B. C. Pal, and I. Erlich, "Impact of wakes on wind farm inertial response," IEEE Transactions on Sustainable Energy, vol. 5, no. 1, pp. 237-245, 2014.

[30] M. Samorani, "The wind farm layout optimization problem," in Handbook of wind power systems. Springer, 2013, pp. 21-38.

[31] R. Kempener and E. Borden, "Battery storage for renewables: Market status and technology outlook," International Renewable Energy Agency, Abu Dhabi, 2015.

[32] A. A. Akhil, G. Huff, A. B. Currier, B. C. Kaun, D. M. Rastler, S. B. Chen, A. L. Cotter, D. T. Bradshaw, and W. D. Gauntlett, "DOE/EPRI electricity storage handbook in collaboration with NRECA," Sandia National Laboratories, SAND2015-1002, 2015.

[33] D. G. Newnan, T. Eschenbach, and J. P. Lavelle, Engineering Economic Analysis, 13th ed. Oxford University Press, 2017.

[34] A. Emami and P. Noghreh, "New approach on optimization in placement of wind turbines within wind farm by genetic algorithms," Renewable Energy, vol. 35, no. 7, pp. 1559-1564, 2010.

[35] Y. Chen, H. Li, K. Jin, and Q. Song, "Wind farm layout optimization using genetic algorithm with different hub height wind turbines," Energy Conversion and Management, vol. 70, pp. 56-65, 2013.

[36] A. Kusiak and Z. Song, "Design of wind farm layout for maximum wind energy capture," Renewable Energy, vol. 35, no. 3, pp. 685-694, 2010.

[37] "Marine data exhchange, the crown estate." [Online]. Available: http://www.marinedataexchange.co.uk/wind-data.aspx

[38] B. Cleary, A. Duffy, A. OConnor, M. Conlon, and V. Fthenakis, "Assessing the economic benefits of compressed air energy storage for mitigating wind curtailment," IEEE Transactions on Sustainable Energy, vol. 6, no. 3, pp. 1021-1028, 2015.

[39] D. J. Burke and M. J. O'Malley, "Factors influencing wind energy curtailment," IEEE Transactions on Sustainable Energy, vol. 2, no. 2 , pp. 185-193, 2011.

[40] H. Li, Z. Lu, Y. Qiao, and N. Wang, "A non-sequential probabilistic production simulation method for wind energy curtailment evaluation considering the seasonal heat supply constraints," IEEE Transactions on Sustainable Energy, vol. 9, no. 1, pp. 462-473, 2018.

[41] L. Castro-Santos, A. Filgueira-Vizoso, I. Lamas-Galdo, and L. CarralCouce, "Methodology to calculate the installation costs of offshore wind farms located in deep waters," Journal of Cleaner Production, vol. 170, pp. 1124-1135, 2018. 